\title{
Reduced resilience of functional state transitions in patients with anti-NMDA receptor encephalitis
}

Nina von Schwanenflug ${ }^{1,2}$, Juan P Ramirez-Mahaluf ${ }^{3}$, Stephan Krohn ${ }^{1,2}$, Amy Romanello ${ }^{1,2}$, Josephine Heine $^{1}$, Harald Prüss ${ }^{1,4}$, Nicolas A Crossley ${ }^{3,5,6,7}$, Carsten Finke ${ }^{1,2}$

\section{Author affiliations:}

${ }^{1}$ Department of Neurology, Charité - Universitätsmedizin Berlin, Corporate Member of Freie Universität Berlin, Humboldt-Universität zu Berlin, and Berlin Institute of Health, Berlin, Germany

${ }^{2}$ Berlin School of Mind and Brain, Humboldt-Universität zu Berlin, Berlin, Germany

${ }^{3}$ Department of Psychiatry, School of Medicine, Pontificia Universidad Católica de Chile, Santiago, Chile

${ }^{4}$ German Centre for Neurodegenerative Diseases, DZNE, Berlin, Germany

${ }^{5}$ Biomedical Imaging Center, Pontificia Universidad Católica de Chile

${ }^{6}$ Millennium Nucleus for Cardiovascular Magnetic Resonance, Chile

${ }^{7}$ Institute of Psychiatry, Psychology and Neuroscience, King's College London, London UK

Correspondence to: Carsten Finke

address: Charitéplatz 1, 10117 Berlin, Germany;

email: carsten.finke@charite.de

Word count: 3486 words

Tables: 1

Figures: $\mathbf{3}$ 


\section{ABSTRACT}

Background: Patients with anti-NMDA receptor (NMDAR) encephalitis suffer from a severe neuropsychiatric syndrome, yet most patients show no abnormalities in routine magnetic resonance imaging (MRI). In contrast, advanced neuroimaging studies have consistently identified disrupted functional connectivity in these patients, with recent work suggesting increased volatility of functional state dynamics. Here, we investigate these network dynamics through the spatiotemporal trajectory of meta-state transitions, yielding a time-resolved account of brain state exploration in NMDAR encephalitis.

Methods: Resting-state functional MRI data were acquired in 73 patients with NMDAR encephalitis and 73 age- and sex-matched healthy controls. Time-resolved functional connectivity was clustered into brain meta-states, giving rise to a time-resolved transition network graph with states as nodes and transitions between brain meta-states as weighted, directed edges. Network topology, robustness, and transition cost of these transition networks were compared between groups.

Results: Transition networks of patients showed significantly lower local efficiency $(t=-2.43, p=$ $0.016)$, lower robustness ( $t=-2.03, p=0.044)$ and higher leap size $(t=2.21, p=0.029)$ compared to controls. Furthermore, the ratio of within-to-between module transitions and state similarity was significantly lower in patients. Importantly, these metrics were correlated with disease severity and disease duration.

Conclusions: These findings reveal systematic alterations of transition networks in patients, suggesting that NMDAR encephalitis is characterized by reduced stability of brain state transitions and that this reduced resilience of transition networks plays a clinically relevant role in the manifestation of the disease.

Keywords: autoimmune encephalitis, MRI, functional imaging, NMDA 


\section{INTRODUCTION}

Anti- $N$-methyl-aspartate receptor (NMDAR) encephalitis is an immune-mediated disorder of the central nervous system caused by autoantibodies targeting the NMDA receptor and leading to a dysregulation of the glutamatergic neurotransmitter system.[1] The disease manifests in a complex neuropsychiatric syndrome with seizures, dyskinesias, psychosis, decreased levels of consciousness, and cognitive dysfunction.[2,3] Despite the severe disease course, only $50-70 \%$ of patients show abnormalities in standard structural magnetic resonance imaging (MRI),[3,4] resulting in a clinicoradiological paradox. In contrast, several functional MRI studies have suggested disrupted functional connectivity (FC) in NMDAR encephalitis that is linked to disease severity, disease duration and cognitive symptoms.[2,5-7]

FC as measured with resting-state functional MRI (rs-fMRI) is estimated from the pairwise correlation of blood oxygen level-dependent (BOLD) activity between brain regions without the presence of an explicit task.[8] However, traditional 'static' approaches obtain FC as an average across several minutes, therefore missing important information that may be derived from dynamic changes in functional connections. $[9,10]$ Hence, the analysis of FC has been recently refined from a time-invariant static account to a time-varying description. This methodological progress allows to unveil temporal properties of functional brain organization, such as the identification of functional states, i.e., transient connectivity patterns, and their transition trajectories. These FC dynamics are thought to reflect brain state exploration that facilitates cognition and behavior, and may vary with disease.[11-13] Accordingly, a recent case-control study investigating FC dynamics in NMDAR encephalitis showed that patients exhibited altered state preference as well as increased transition frequencies between major connectivity patterns.[14] However, a detailed investigation of the transition trajectory of brain states and its link to clinical symptoms is still missing. Brain state exploration - facilitated by transitions between functional states - is thought to ensure stable information representation while promoting functional integration across distant brain regions and subsystems and, if disturbed, potentially affects information integration, behavior, and cognitive performance.[12,15] Hence, identifying mechanisms and disruptions of these transition trajectories may contribute to the understanding of the pathophysiology of the disease.

Graph theoretical approaches are well-suited to study the temporal architecture of state exploration. Ramirez-Mahaluf et al. [16] recently introduced the concept of transition networks to investigate the trajectory of traversing functional states (from hereon also referred to as meta-states following [16]). In this concept, transition networks are represented as graphs with brain states as nodes and transitions between meta-states as directed and weighted edges. Similar to other biological systems,[17] transition networks show properties of complex networks (i.e., heavy-tailed degree 
distribution, high local efficiency, and modularity) indicating an organized, cost-efficient, non-random temporal trajectory of brain states.[16] Furthermore, transition network characteristics have been related to motor function and cognitive performance in healthy controls indicating behavioral relevance.[16]

Here, we aimed to specify alterations of the spatiotemporal trajectory of state transitions and its relation to disease severity in NMDAR encephalitis. Therefore, we constructed transition networks for a large sample of patients and age- and sex-matched healthy controls. We hypothesized that the temporal structure of state exploration in NMDAR encephalitis would show altered dynamics and weakened stability of transition networks compared to a group of healthy controls. 


\section{MATERALS AND METHODS}

\section{Participants}

For this study, 73 patients with NMDAR encephalitis were recruited from the Department of Neurology at Charité - Universitätsmedizin Berlin. All patients fulfilled diagnostic criteria including characteristic clinical presentation and detection of IgG NMDA receptor antibodies in the cerebrospinal fluid.[3] Patients were in the post-acute phase of their disease with a median of 2.97 years (IQR: 2.48) after disease onset. Disease severity at the time of scan and peak of disease was assessed with the modified Rankin scale (mRS). The control group consisted of 73 age- and sex-matched healthy participants without any history of neurological or psychiatric disease. Patient-control matching was optimized for age and sex through a computational matching algorithm (see Supplemental Material 1). The two groups were perfectly balanced for sex and did not differ significantly in age as tested with a Wilcoxon rank sum test $(p=0.61)$. Clinical and demographic characteristics are summarized in Table 1.

Table 1: Demographic variables and clinical measures of the participants. Table lists median and interquartile range (IQR) of age, $m R S$ at scan, $m R S$ at peak of disease, disease duration, and time between scan and diagnosis. Treatment and medication during disease course were evaluated using a binary scale (present: 'yes' vs. absent: ' $n o$ '). Disease duration = hospitalization in days during acute phase of the disease; $\mathrm{N}=$ number of participants; $\underline{\mathrm{mRS}}=$ modified Rankin Scale.

\begin{tabular}{cccc}
\hline & & $\begin{array}{c}\text { NMDAR encephalitis } \\
\text { Patients }\end{array}$ & Healthy Controls \\
\hline $\mathbf{N}$ & & 73 & 73 \\
Sex & female/male & $62 / 11$ & $62 / 11$ \\
Age (years) & Median \pm IQR (N) & $25.68 \pm 12.60(73)$ & $26.40 \pm 14.09(73)$ \\
mRS at scan & Median \pm IQR (N) & $1.00 \pm 1.5(70)$ &.. \\
time) & Median \pm IQR (N) & $67.50 \pm 72.00(68)$ &.. \\
Disease duration (hospitalization & & &.. \\
Years between disease onset and & Mean \pm SD (N) & $2.97 \pm 2.48(71)$ &.. \\
study & & &.. \\
First-line treatment & yes/no & $72 / 1$ &.. \\
Second-line treatment & yes/no & $37 / 36$ &.. \\
Anticonvulsant medication & yes/no & $51 / 22$ & \\
Antipsychotic medication & yes/no & $48 / 25$ & \\
\hline
\end{tabular}

\section{MRI data acquisition}

MRI data was collected at the Berlin Center for Advanced Neuroimaging at Charité Universitätsmedizin Berlin using a 3T Trim Trio scanner equipped with a 20-channel head coil (Siemens, Erlangen, Germany). Resting-state functional images were acquired using an echoplanar imaging sequence $\left(T R=2.25 \mathrm{~s}, T E=30 \mathrm{~ms}, 260\right.$ volumes, voxel size $\left.=3.4 \times 3.4 \times 3.4 \mathrm{~mm}^{3}\right)$. High-resolution T1weighted structural scans were collected using a magnetization-prepared rapid gradient echo sequence (MPRAGE; voxel size $=1 \times 1 \times 1 \mathrm{~mm}^{3}$ ). 


\section{MRI data analysis}

Prior to preprocessing, framewise displacement (FD) was calculated for each participant and assessed against a mean FD cutoff of $0.50 \mathrm{~mm}$.[18,19] No participant had a mean FD greater than or equal to $0.50 \mathrm{~mm}$. Preprocessing was performed using customized MATLAB scripts based on Parkes et al. [20] and included removal of the first 4 volumes of each participant's rs-fMRI scan, slice-timing correction, detrending of BOLD time-series, intensity normalization, and spatial smoothing with $6 \mathrm{~mm}$ full width at half maximum. Motion correction was performed using ICA-AROMA.[21] Additionally, motion parameters, mean white matter and CSF time-series were regressed out during denoising. Finally, bandpass filtering to retain frequencies between 0.008 and $0.08 \mathrm{~Hz}$ and demeaning were performed. Timeseries extraction was done using a whole-brain parcellation template with 638 regions of interests (ROI). [22]

\section{Participant-wise meta-state estimation and transition network construction}

Data were processed as previously described in Ramirez-Mahaluf et al (2020).[16] Functional timeseries were segmented into 127 consecutive time windows of 2TRs ( $\triangleq 4.5$ seconds). For each window, FC was estimated between any two ROIs using Multiplicaton of Temporal Derivatives.[23] The resulting ROI-by-ROI (638-by-638) matrices were then Pearson-correlated, resulting in a 127-by-127 similarity matrix of windows. To obtain discrete brain meta-states MATLAB-inbuilt k-means clustering was applied to the similarity matrix using 10000 maximum iterations and 2000 replicates with random initial positions. For each meta-state, all windows belonging to that state are averaged, yielding a mean ROI-by-ROI (638-by-638) connectivity matrix. Following Ramirez-Mahaluf et al. (2020), we extracted multiple $k$ meta-states $(35,40,45,50$, and 55$)$ for each participant separately. [16]

Transition networks for each $k$ were represented as directed graphs, with each meta-state corresponding to a node and the number of transitions between meta-states corresponding to directed weighted edges.[16]

\section{Group comparisons of transition network properties}

We assessed group differences of three widely used graph theoretical measures (modularity, local efficiency, and global efficiency), as well as two custom measures that are thought to capture the biological costs of meta-state transitions (leap size and immobility).[16] Note that modularity, local and global efficiency, and immobility were calculated on the transition matrix for each participant, while leap size was based on the distance matrix (i.e., 1-correlation for each meta-state pair). 
Furthermore, the robustness of the transition networks against perturbation was assessed for each participant. Here, we employed the NetSwan package available for $R$ to randomly remove one node after another from the network and recalculate the size of the largest connected component.[24-26] A more detailed description of the graph theoretical metrics is provided in Supplemental Table 1.

In addition, transition frequency, ratio of within-to-between module meta-state similarity, and ratio of within-to-between module transitions were compared between patients and controls. Here, a 'module' refers to a group of meta-states assigned to the same community as defined by the modularity algorithm (community_louvain.m). While transition frequency is calculated as the absolute number of transitions between different meta-states, the ratio of within-to-between meta-state similarity $\left(\right.$ ratio $_{\text {sim }}$ ) is defined as the average correlation of meta-states within a module divided by the average correlation of meta-states between modules. Similarly, the ratio of within-to-between module transitions (ratio trans $_{\text {) }}$ is the absolute number of transitions within the same module divided by the absolute number of transitions between modules.

Between-group comparisons of graph theoretical measures, transition frequencies, ratio $_{\text {sim }}$, and ratio $_{\text {trans }}$ were assessed by calculating the area under the curve (AUC) for all $k$ number of meta-states for each metric and participant. The AUC was standardized across participants (zscore) and entered into a general linear model (MATLAB's glmfit) with framewise displacement, age, and sex as nuisance variables for each metric separately. Network metrics were not corrected for multiple comparison.

\section{Functional network topology of meta-states}

Lastly, we evaluated the region-wise difference in functional topology across all meta-states to assess which regions primarily promote the state switches. Due to the high number of meta-states, we assessed a summary measure for each participant, which is the cumulative Euclidean distance for every edge in the ROI-by-ROI connectivity matrices across meta-state space. This resulted in a single ROI-byROI matrix representing the across-state-distance (ASD) for each participant and $k$ number of metastates as defined previously.[27] A high value of ASD between $\mathrm{ROI}_{\mathrm{i}}$ and $\mathrm{ROI}_{\mathrm{j}}$ indicates that the connectivity between these regions differs strongly between meta-states. In contrast, a low ASD indicates that the connectivity between these regions is similar across all meta-states of a transition network.

For each participant, ASD maps for each $k$ were averaged and group differences for each edge were assessed with a two-sample t-test and corrected for multiple comparisons ( $n=203203$ edges). 


\section{RESULTS}

\section{Group differences in network properties}

Group comparisons of graph theoretical measures yielded significantly lower local efficiency (std. $B=$ $-0.12, t=-2.43, p=0.016$ ), higher leap size (std. $b=0.18, t=2.21, p=0.029)$, and lower robustness (std. $B=-0.17, t=-2.03, p=0.044)$ of transition networks in patients compared to controls. In contrast, modularity (std. $b=-0.12, t=-1.44, p=0.15$ ), global efficiency (std. $b=0.085, t=1.018, p=0.31$ ), and immobility (std. $B=-0.028, t=-0.33, p=0.74$ ) of transitions networks did not differ between groups (Fig. 1). Supplemental Fig. 2 shows the transition networks of six exemplary participants with high and low leap size.
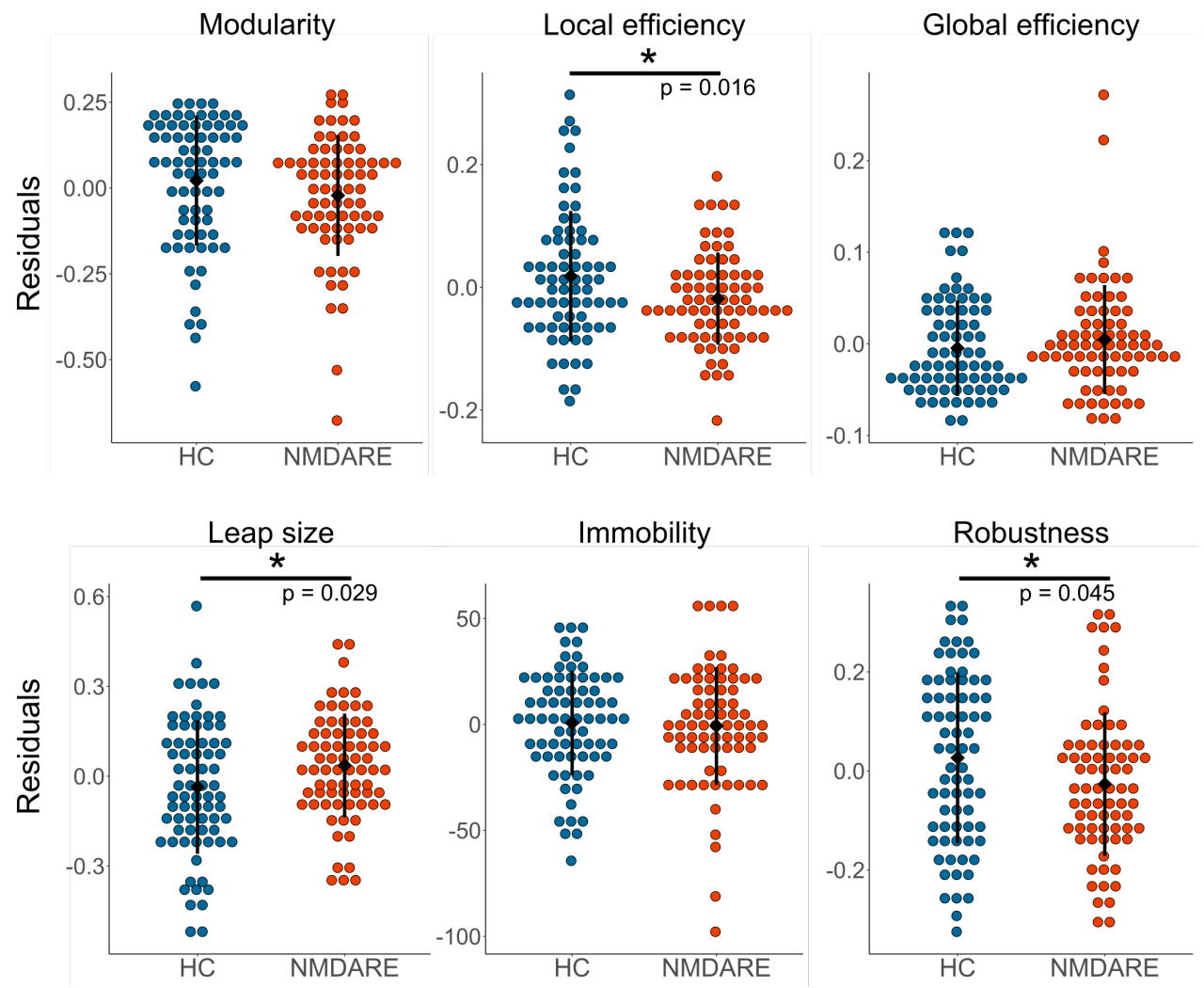

Figure 1: Between-group comparisons of graph theoretical measures. Colored dots represent the residuals from the general linear model. Black dots and whiskers represent the mean and standard deviation, respectively. $\mathrm{HC}$ = healthy controls, NMDAR encephalitis = patients with anti-NMDA receptor encephalitis. * indicates significant difference $p<0.05$.

Correlation of similarity and the number of transitions between two meta-states revealed that transition frequency was higher between similar meta-states for both groups and all numbers of metastates (rho $=[0.48,0.53,0.54,0.54,0.52]$ for the different $k$ meta-states; all $p<0.001$, Fig. 2 ). Accordingly, transitions within modules were on average 3.4 times more likely than between modules with a ratiotrans (ratio of within-to-between module transitions) $=[2.40,2.94,3.44,3.89,4.44]$ 
depending on the number of meta-states. This result was expected as modularity is calculated on the

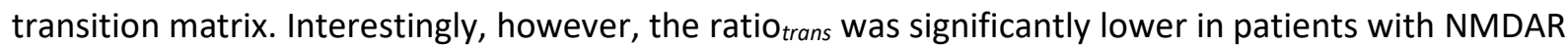
encephalitis compared to controls (std. $B=-0.20, t=-2.46, p=0.015$ ), while the overall number of transitions between different meta-states did not differ between groups (std. $b=0.028, t=0.33, p=$ 0.74). Similar to ratio ${ }_{\text {trans, }}$ ratio $_{\text {sim }}$ (ratio of within-to-between meta-state similarity) was on average 3.2 (ratio $_{\text {sim }}=[2.9,3.1,3.2,3.3,3.4]$, for the different $k$ meta-states). Again, the ratio sim $_{\text {sim }}$ was significantly lower in patients compared to controls (std. $b=-0.21, t=-2.51, p=0.013$ ). This suggests that patients transition between topologically more different meta-states (from different modules) compared to controls, while the overall transition frequency remains unaltered.

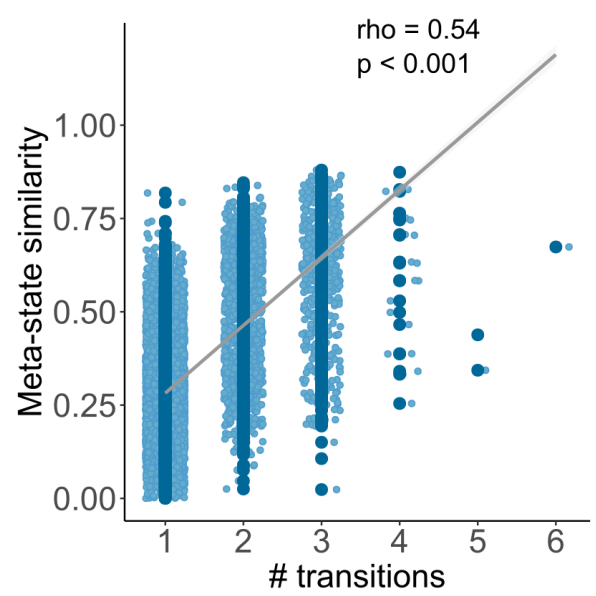

Figure 2: Correlation between meta-state similarity and number of transitions between them (here shown for $k$

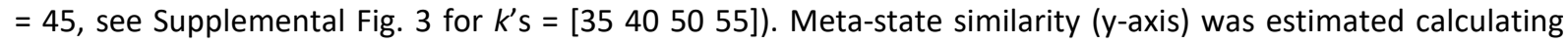
Spearman's $\mathrm{e}$. Number of transitions ( $\mathrm{x}$-axis) are the sum of transitions between any two meta-states, independent of the direction of transitions.

Next, we investigated the relationship of local efficiency, leap size, and robustness with disease severity ( $m R S$ at the time of scan) and acute days in hospitalization. We observed that disease severity was significantly correlated with leap size ( $r h o=0.24, p=0.049$ ), but not with local efficiency ( $r h o=$ $0.10, p=0.399$ ), or robustness ( $r h o=-0.21, p=0.084$ ). Longer disease duration, i.e., acute days in hospitalisation, was significantly associated with lower local efficiency (Pearson's $r=-0.26, p=0.032$ ), higher leap size (Pearson's $r=0.35, p=0.0037$ ), and decreased robustness (Pearson's $r=-0.38, p=$ $0.0014)$.

Additionally, we investigated the relationship of disease severity and disease duration with ratio trans $_{\text {s }}$

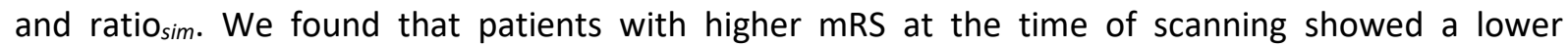
ratio $_{\text {sim }}($ rho $=-0.24, p=0.047)$, wheras no significant correlation was found for ratio trans $_{\text {( }}$ (rho $=-0.174$, 
$p=0.153)$. Furthermore, longer disease duration was significantly associated with ratio ${ }_{s i m}$ (Pearson's $r$ $=-0.41, p<0.001)$ and ratio trans $($ Pearson's $r=-0.32, p=0.0079)$.

\section{Functional network topology of meta-states}

The edges with the highest ASD, i.e., edges that exhibited most pronounced differences over metastates, clustered predominantly in unimodal networks, namely the sensorimotor and visual network (Fig. 3A). This topological pattern is highly convergent with recent findings from Krohn et al.[27]

Whole-brain group comparison yielded no significant difference in ASD between groups after correction for multiple comparison. Yet, exploratory network-wise group comparison revealed significant differences between edges within the visual, default mode and sensorimotor networks (Fig 3B). Remarkably, significant edges showed higher ASD in patients within the visual and sensorimotor network, but lower ASD within the default-mode network (Supplemental Table 3).
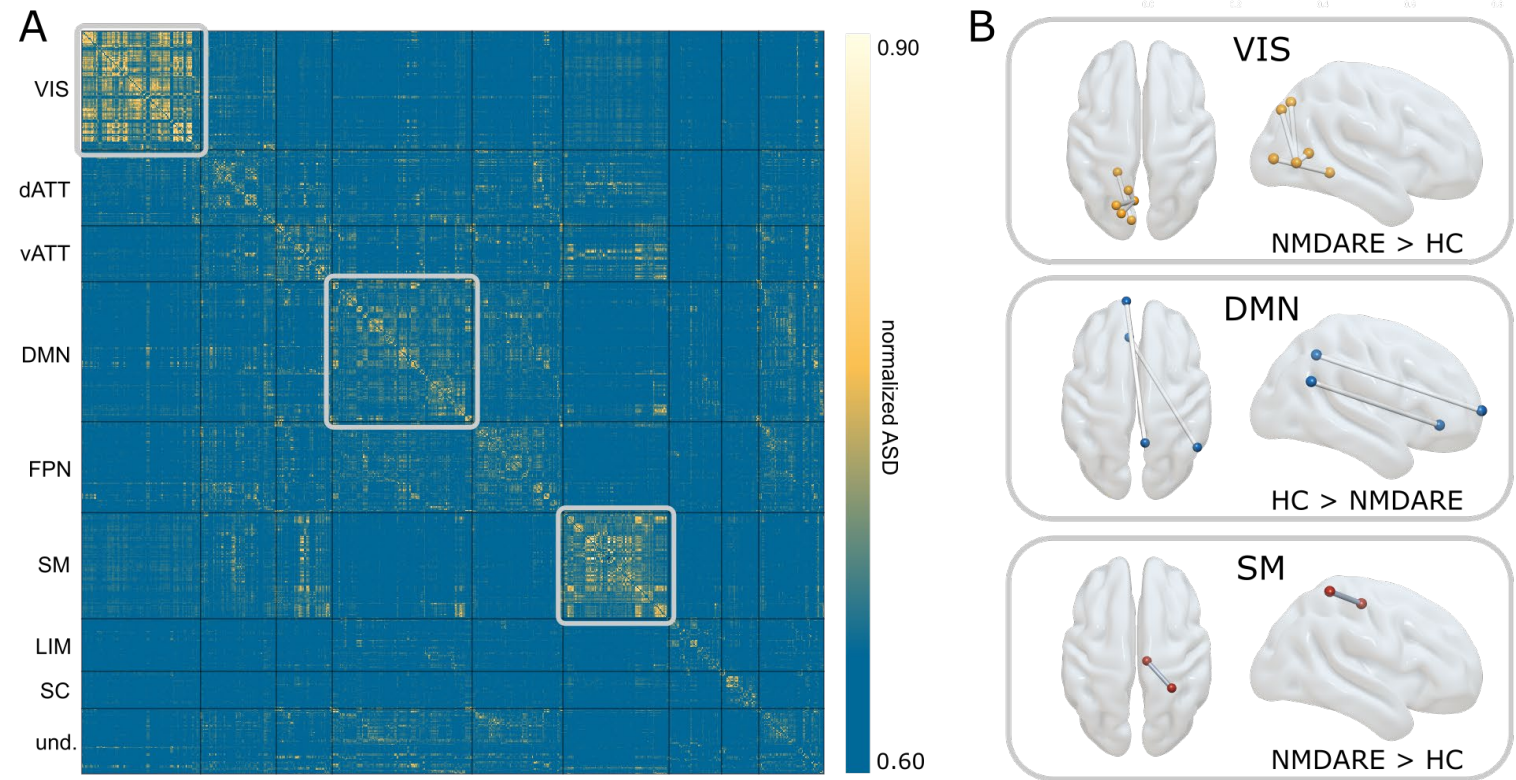

Figure 3: Interregional across-state distance (ASD) across all participants and normalized for the number of metastates. Network assignment of regions is based on the labels proposed by Yeo et al.[28] Subcortical regions were subsumed as a subcortical network. Brain plots show edges that are significantly different between patients and healthy controls within the visual, default-mode and sensorimotor network. VIS = visual network, dATT = dorsal attention network, $\mathrm{VATT}=$ ventral attention network, DMN = default mod network, FPN = fronto-parietal network, SM = sensorimotor network, LIM = limbic network, SC = subcortical network, und. = undefined, $H C=$ healthy controls, NMDAR encephalitis = patients with anti-NMDA receptor encephalitis. 


\section{DISCUSSION}

Ongoing brain activity can be described as transient FC patterns (so-called brain states) that are visited in a structured, non-random trajectory.[16] However, these brain state dynamics can vary in disease, with links to dysfunctions in cognition and behavior.[11] In this study, we employed a time-resolved analysis of brain activity to capture the spatiotemporal dynamics of brain state transitions in a large sample of patients with NMDAR encephalitis. Our results indicate reduced resilience of state transition networks in patients compared to controls. This manifests in lower local efficiency of the network (fewer transitions from or to neighboring, i.e., similar, meta-states), higher leap size (transitions between more distinct meta-states), and reduced robustness of the patients' transition networks against random attacks. Furthermore, the ratio of within-to-between module transitions and metastate similarity was significantly reduced in patients. Importantly, these state dynamic metrics were correlated with disease severity at the time of scanning and/or disease duration, highlighting the clinical relevance of our findings.

In patients with NMDAR encephalitis, autoantibodies target the NR1 subunit of the NMDA receptor causing an internalization of the receptor.[29] While this results in a broad range of psychiatric and neurological symptoms, standard clinical MRI shows no or only minor abnormalities in most patients.[3,4] In contrast, FC analyses were able to identify characteristic connectivity alterations: Static resting-state FC analyses that average connectivity across an entire scanning session showed widespread disrupted connectivity in visual, temporal, hippocampal, and mid-frontal areas associated with the severity of cognitive and psychiatric symptoms.[5,30,31] However, given that brain activity is inherently dynamic,[32] models that incorporate spatiotemporal features of connectivity should even better reflect functional disruptions in neuropsychiatric disorders. Indeed, we recently found that dynamic FC showed a shift in state preference and transition probabilities in patients with NMDAR encephalitis that was associated with disease severity and disease duration.[14] In the present study, we further expand on these dynamic FC findings and investigated alterations in the spatiotemporal trajectory of functional state exploration through the underlying state space. State exploration is thought to reflect the dynamic repertoire of intrinsic brain activity that is important for information integration and mental processes.[12,15,33] Therefore, disruptions in the temporal organization of state transitions may account for behavioral and clinical symptoms in disease.[11,34] In fact, we found a characteristic spatiotemporal reorganization of the transition trajectory in patients compared to controls that was related to disease severity. This spatiotemporal reorganization - as reflected by lower local efficiency, lower robustness, and higher leap size of the transition network - may represent overly unstable transition dynamics that could be linked to deficiencies in information integration.[15,34] 
At a scale of seconds to minutes, the human brain operates through continuously evolving activity that can be characterized as transient quasi-stable brain states. $[9,10]$ This evolution of brain activity is nonrandom, allowing for a systematic exploration of brain states.[16] Analogous to the modular spatial organization of the cortex, the temporal trajectory of brain state transitions shows similar topological properties, i.e. brain states are grouped into modules of similar meta-states, with higher transition frequencies within a module than between modules (see methods and results section: ratio trans $_{/} /$ratio $_{\text {sim }}$ ). This modular organization is thought to promote segmented and cost-efficient information processing, while enabling the exploration of the functional repertoire via transitions to meta-states of a different module.[13,34-37] In line with the modular structure, transition networks in healthy controls show high local efficiency, and low global efficiency (as compared to a null model).[16] While a high local efficiency allows for locally specialized brain functioning, a comparatively smaller number of connections between these networks, i.e., low global efficiency, furthermore allows for distributed information processing across different subsystems. [37] Moreover, a high local efficiency enhances the robustness of a system. By providing alternative pathways between two nodes (i.e., meta-states), the system compensates for potential disturbances and provides stable representation of information.[38] Interestingly, the spatiotemporal organization of state exploration may also be directly relevant to behavior. A recent study on transition networks in a healthy population suggests that the efficiency of the network is associated with performance in cognition and motor function.[16] Thus, state exploration may vary across diseases potentially accounting for a multitude of symptoms.[11]

Indeed, the present study highlights significant differences in the temporal architecture of transition networks between patients with NMDAR encephalitis and healthy controls. We found that patients exhibited decreases in local efficiency and robustness, and increases in leap size. Decreased local efficiency hints at unstable representation of information due to lower redundancy of the transition network, which is described in more detail in the previous paragraph. Leap size is thought to reflect metabolic cost and is measured as the magnitude of 'jumps' between topologically different metastates. Eliciting state transitions is energetically costly, $[33,39]$ and possibly increases when traversing states that show highly disparate activation profiles. This intuition is supported by our finding that (low-cost) transitions between two similar meta-states are more likely than (cost-intensive) transitions between distant meta-states. Accordingly, higher leap size in patients may indicate higher metabolic demand along with higher volatility of state transitions. Lastly, we evaluated the robustness of the transition network, which is the ability of maintaining information processing within the network before collapsing.[40] We found that transition networks of patients with NMDAR encephalitis are less robust compared to those of controls when removing the nodes (i.e., meta-states) one by one. Together with a decreased local efficiency and higher leap size, this points to a destabilization and 
reduced resilience of transition networks in patients with NMDAR encephalitis. This notion is furthermore supported by decreased ratios of within-to-between module transitions and within-tobetween module meta-state similarity in patients. In addition, all five network measures - local efficiency, leap size, robustness, ratio $_{\text {trans, }}$ and ratio $_{\text {sim }}$ - were correlated with disease severity at the time of scan and/or disease duration, supporting clinical relevance of our findings. Interestingly, reduced resilience of transition networks in patients with NMDAR encephalitis is supported by findings from attractor-based computational models that postulate that NMDAR dysfunction may lead to overly unstable attractors in brain activity.[41,42] NMDAR hypofunction, as in NMDAR encephalitis, may lead to a flattening of the attractors (destabilizing effect), facilitating perturbations to provoke transitions between attractors.[41]

Finally, our study provides evidence that a subset of regions preferentially promotes brain state transitions.[11,27] Convergent with recent work on brain dynamics, we found that changes in connectivity across states are most pronounced in regions of the visual and sensorimotor areas, potentially following a hierarchy from unimodal to transmodal networks.[27] Interestingly, in patients with NMDAR encephalitis, connectivity changes across meta-states within unimodal networks were even more pronounced, whereas connectivity between regions within the default-mode networks was attenuated. Limiting state transitions to a defined number of regions initiating those transitions raises the intriguing possibility that controlled external stimulation of these particular regions could be applied to achieve a rebalancing of state dynamics.[11,33]

Some limitations of our study deserve mentioning: First, the window length of 2 TR ( $\hat{=} 4.5$ seconds) is comparatively short and may decrease the signal-to-noise ratio. However, a high reliability of metastate estimation was previously shown using a similar window length.[16] Second, k-means clustering is applied to each participant separately, which may lead to different topologies of meta-states across participants. As a consequence, the transition network approach is well-suited to investigate characteristics of the transition trajectory of functional states, however, between-group comparison of the topology of each single state is limited. Third, the applied $k$ enforces the extraction of a large number of (potentially similar) meta-states for each participant. While most studies focus on 3-5 distinct major connectivity states, this number of states may not be sufficient to represent the full repertoire of functional configurations of the human brain. Furthermore, a small number of states limits a detailed investigation of transition trajectories between these states, which was the main purpose of the present study.

\section{Conclusion}

In this study, we employed a time-resolved graph analytical framework to study the spatiotemporal trajectory of brain state transitions in patients with NMDAR encephalitis. Besides decreases in local 
efficiency, we observed reduced robustness of the patients' transition networks against random attacks compared to those of healthy controls. Together with higher leap size in patients, these findings show reduced resilience of functional state transitions in patients, which is related to disease duration and severity. Hence, our findings add to the evidence that disturbance of functional brain network dynamics plays a key role in the pathophysiology of NMDAR encephalitis. 


\section{AUTHOR CONTRIBUTIONS}

NS and CF designed the study, interpreted the results. CF, JH, HP conceived the cohort study and data collection. NS conducted the analyses, wrote the first draft of the study and produced the figures. JRM developed the graph transition network construction and provided analysis code. SK implemented the across-state-distance and the matching algorithm. JRM, NAC and SK significantly contributed to the design and interpretation of the analysis. AR implemented the preprocessing pipeline All authors edited and revised the manuscript.

\section{FUNDING}

Funded by the Deutsche Forschungsgemeinschaft (DFG, German Research Foundation, grant numbers 327654276 (SFB 1315), FI 2309/1-1 and FI 2309/2-1) and Deutsches Ministerium für Bildung und Forschung (BMBF, German Ministry of Education and Research, grant number 01GM1908D, CONNECTGENERATE). NS is a doctoral scholar at Cusanuswerk - Bischöfliche Studienförderung. AR is a doctoral scholar at the Berlin School of Mind and Brain, Humboldt-Universität zu Berlin. JRM and NAC were funded by the Agencia Nacional de Investigación y Desarrollo from Chile (ANID), through its programs FONDECYT postdoctorado (Ref: 3190311 to JRM), FONDECYT regular (Ref: 1200601 to NAC), and Anillo ACT192064. The funders had no influence on study design, data collection, data analyses, data interpretation, or writing.

\section{COMPETING INTERSTS}

NS, JRM, SK, AR, JH, HP, and CF have no competing interests. NAC has received personal fees from Janssen (Johnson \& Johnson), outside the submitted work.

\section{SUPPLEMENTAL MATERIAL}

Supplemental material is available online.

\section{ETHICS STATEMENTS}

\section{Patient consent for publication}

Not applicable.

\section{Ethics approval}

The study was approved by the ethics committee of the Charité - Universitätsklinikum Berlin and all participants gave written informed consent before participation.

\section{DATA AVAILABILITY}


bioRxiv preprint doi: https://doi.org/10.1101/2022.01.24.477081; this version posted January 24, 2022. The copyright holder for this preprint (which was not certified by peer review) is the author/funder. All rights reserved. No reuse allowed without permission.

With respect to the sensitivity of the data, we provide de-identified data and the code that support the presented findings upon reasonable request, subject to a material transfer agreement. 


\section{REFERENCES}

1 Dalmau J, Armangué T, Planagumà J, et al. An update on anti-NMDA receptor encephalitis for neurologists and psychiatrists: mechanisms and models. Lancet Neurol 2019;18:1045-57. doi:10.1016/S1474-4422(19)30244-3

2 Finke $\mathrm{C}$, Kopp UA, Prüss $\mathrm{H}$, et al. Cognitive deficits following anti-NMDA receptor encephalitis. J Neurol Neurosurg Psychiatry 2012;83:195-8. doi:10.1136/jnnp-2011-300411

3 Graus F, Titulaer MJ, Balu R, et al. A clinical approach to diagnosis of autoimmune encephalitis. Lancet Neurol 2016;15:391-404. doi:10.1016/S1474-4422(15)00401-9

4 Heine J, Prüss H, Bartsch T, et al. Imaging of autoimmune encephalitis - Relevance for clinical practice and hippocampal function. Neuroscience 2015;309:68-83.

doi:10.1016/j.neuroscience.2015.05.037

5 Finke C, Kopp UA, Scheel M, et al. Functional and structural brain changes in anti-N-methyl-Daspartate receptor encephalitis. Ann Neurol 2013;74:284-96.

6 Gibson LL, Pollak TA, Blackman G, et al. The Psychiatric Phenotype of Anti-NMDA Receptor Encephalitis. J Neuropsychiatry Clin Neurosci 2019;31:70-9.

doi:10.1176/appi.neuropsych.17120343

7 Gibson LL, McKeever A, Coutinho E, et al. Cognitive impact of neuronal antibodies: encephalitis and beyond. Transl Psychiatry 2020;10:304. doi:10.1038/s41398-020-00989-x

8 Biswal B, Zerrin Yetkin F, Haughton VM, et al. Functional connectivity in the motor cortex of resting human brain using echo-planar mri. Magn Reson Med 1995;34:537-41. doi:10.1002/mrm.1910340409

9 Allen EA, Damaraju E, Plis SM, et al. Tracking whole-brain connectivity dynamics in the resting state. Cereb Cortex N Y N 1991 2014;24:663-76. doi:10.1093/cercor/bhs352

10 Calhoun VD, Miller R, Pearlson G, et al. The Chronnectome: Time-Varying Connectivity Networks as the Next Frontier in fMRI Data Discovery. Neuron 2014;84:262-74.

doi:10.1016/j.neuron.2014.10.015

11 Kringelbach ML, Deco G. Brain States and Transitions: Insights from Computational Neuroscience. Cell Rep 2020;32:108128. doi:10.1016/j.celrep.2020.108128

12 Deco G, Jirsa VK, McIntosh AR. Emerging concepts for the dynamical organization of resting-state activity in the brain. Nat Rev Neurosci 2011;12:43-56. doi:10.1038/nrn2961

13 Bassett DS, Wymbs NF, Porter MA, et al. Dynamic reconfiguration of human brain networks during learning. Proc Natl Acad Sci 2011;108:7641-6. doi:10.1073/pnas.1018985108

14 von Schwanenflug N, Krohn S, Heine J, et al. State-dependent signatures of Anti-NMDA-Receptor Encephalitis: a dynamic functional connectivity study. Brain Communications 2022; in press.

15 Lord L-D, Expert P, Atasoy S, et al. Dynamical exploration of the repertoire of brain networks at rest is modulated by psilocybin. Neurolmage 2019;199:127-42.

doi:10.1016/j.neuroimage.2019.05.060 
16 Ramirez-Mahaluf JP, Medel V, Tepper Á, et al. Transitions between human functional brain networks reveal complex, cost-efficient and behaviorally-relevant temporal paths. Neurolmage 2020;219:117027. doi:10.1016/j.neuroimage.2020.117027

17 Latora V, Marchiori M. Efficient Behavior of Small-World Networks. Phys Rev Lett 2001;87:198701. doi:10.1103/PhysRevLett.87.198701

18 Power JD, Mitra A, Laumann TO, et al. Methods to detect, characterize, and remove motion artifact in resting state fMRI. Neurolmage 2014;84:320-41.

doi:10.1016/j.neuroimage.2013.08.048

19 Eijlers AJC, Wink AM, Meijer KA, et al. Reduced Network Dynamics on Functional MRI Signals Cognitive Impairment in Multiple Sclerosis. Radiology 2019;292:449-57.

doi:10.1148/radiol.2019182623

20 Parkes L, Fulcher B, Yücel M, et al. An evaluation of the efficacy, reliability, and sensitivity of motion correction strategies for resting-state functional MRI. Neurolmage 2018;171:415-36. doi:10.1016/j.neuroimage.2017.12.073

21 Pruim RHR, Mennes M, van Rooij D, et al. ICA-AROMA: A robust ICA-based strategy for removing motion artifacts from fMRI data. Neurolmage 2015;112:267-77.

doi:10.1016/j.neuroimage.2015.02.064

22 Crossley NA, Mechelli A, Vertes PE, et al. Cognitive relevance of the community structure of the human brain functional coactivation network. Proc Natl Acad Sci 2013;110:11583-8. doi:10.1073/pnas.1220826110

23 Shine JM, Koyejo O, Bell PT, et al. Estimation of dynamic functional connectivity using Multiplication of Temporal Derivatives. Neurolmage 2015;122:399-407. doi:10.1016/j.neuroimage.2015.07.064

24 Lynall M-E, Bassett DS, Kerwin R, et al. Functional Connectivity and Brain Networks in Schizophrenia. J Neurosci 2010;30:9477-87. doi:10.1523/JNEUROSCI.0333-10.2010

25 Achard S. A Resilient, Low-Frequency, Small-World Human Brain Functional Network with Highly Connected Association Cortical Hubs. J Neurosci 2006;26:63-72. doi:10.1523/JNEUROSCI.387405.2006

26 Lhomme S. Analyse spatiale de la structure des réseaux techniques dans un contexte de risques. Cybergeo Published Online First: 20 February 2015. doi:10.4000/cybergeo.26763

27 Krohn S, von Schwanenflug N, Waschke L, et al. A spatiotemporal complexity architecture of human brain activity. biorxiv 2021. doi:10.1101/2021.06.04.446948

28 Thomas Yeo BT, Krienen FM, Sepulcre J, et al. The organization of the human cerebral cortex estimated by intrinsic functional connectivity. J Neurophysiol 2011;106:1125-65. doi:10.1152/jn.00338.2011

29 Dalmau J, Lancaster E, Martinez-Hernandez E, et al. Clinical experience and laboratory investigations in patients with anti-NMDAR encephalitis. Lancet Neurol 2011;10:63-74. doi:10.1016/S1474-4422(10)70253-2 
30 Cai L, Liang $\mathrm{Y}$, Huang $\mathrm{H}$, et al. Cerebral functional activity and connectivity changes in anti-Nmethyl-D-aspartate receptor encephalitis: A resting-state fMRI study. Neurolmage Clin 2020;25:102189. doi:10.1016/j.nicl.2020.102189

31 Peer M, Prüss H, Ben-Dayan I, et al. Functional connectivity of large-scale brain networks in patients with anti-NMDA receptor encephalitis: an observational study. Lancet Psychiatry 2017;4:768-74. doi:10.1016/S2215-0366(17)30330-9

32 Chang $\mathrm{C}$, Glover GH. Time-frequency dynamics of resting-state brain connectivity measured with fMRI. Neurolmage 2010;50:81-98. doi:10.1016/j.neuroimage.2009.12.011

33 Gu S, Betzel RF, Mattar MG, et al. Optimal trajectories of brain state transitions. Neurolmage 2017;148:305-17. doi:10.1016/j.neuroimage.2017.01.003

34 Deco G, Kringelbach ML, Jirsa VK, et al. The dynamics of resting fluctuations in the brain: metastability and its dynamical cortical core. Sci Rep 2017;7:3095. doi:10.1038/s41598-01703073-5

35 Tognoli E, Kelso JAS. The Metastable Brain. Neuron 2014;81:35-48. doi:10.1016/j.neuron.2013.12.022

36 Bertolero MA, Yeo BTT, D'Esposito M. The modular and integrative functional architecture of the human brain. Proc Natl Acad Sci 2015;112:E6798-807. doi:10.1073/pnas.1510619112

37 Sporns O, Betzel RF. Modular Brain Networks. Annu Rev Psychol 2016;67:613-40. doi:10.1146/annurev-psych-122414-033634

38 De Vico Fallani F, Aparecido RF, Da Fontoura CL, et al. Analysis of the connection redundancy in functional networks from high-resolution EEG: A preliminary study. In: 2009 Annual International Conference of the IEEE Engineering in Medicine and Biology Society. Minneapolis, MN: : IEEE 2009. 2204-7. doi:10.1109/IEMBS.2009.5334882

39 Lord L-D, Expert P, Huckins JF, et al. Cerebral Energy Metabolism and the Brain's Functional Network Architecture: An Integrative Review. J Cereb Blood Flow Metab 2013;33:1347-54. doi:10.1038/jcbfm.2013.94

40 Aerts $\mathrm{H}$, Fias W, Caeyenberghs $\mathrm{K}$, et al. Brain networks under attack: robustness properties and the impact of lesions. Brain 2016;139:3063-83. doi:10.1093/brain/aww194

41 Rolls ET. Glutamate, obsessive-compulsive disorder, schizophrenia, and the stability of cortical attractor neuronal networks. Pharmacol Biochem Behav 2012;100:736-51. doi:10.1016/j.pbb.2011.06.017

42 Rolls ET. Attractor cortical neurodynamics, schizophrenia, and depression. Transl Psychiatry 2021;11:215. doi:10.1038/s41398-021-01333-7 